\title{
Black holes in astrophysics
}

\section{Citation}

Narayan, Ramesh. 2005. “Black Holes in Astrophysics.” New Journal of Physics 7 (September): 199-199. https://doi.org/10.1088/1367-2630/7/1/199.

\section{Permanent link}

http://nrs.harvard.edu/urn-3:HUL.InstRepos:41384889

\section{Terms of Use}

This article was downloaded from Harvard University's DASH repository, and is made available under the terms and conditions applicable to Other Posted Material, as set forth at http:// nrs.harvard.edu/urn-3:HUL.InstRepos:dash.current.terms-of-use\#LAA

\section{Share Your Story}

The Harvard community has made this article openly available.

Please share how this access benefits you. Submit a story.

Accessibility 


\title{
Black Holes in Astrophysics
}

\author{
Ramesh Narayan \\ Harvard College Observatory, Harvard-Smithsonian Center for Astrophysics, Cambridge, \\ MA 02138, USA \\ rnarayan@cfa.harvard.edu
}

\begin{abstract}
This article reviews the current status of black hole astrophysics, focusing on topics of interest to a physics audience. Astronomers have discovered dozens of compact objects with masses greater than $3 M_{\odot}$, the likely maximum mass of a neutron star. These objects are identified as black hole candidates. Some of the candidates have masses $\sim 5-20 M_{\odot}$ and are found in X-ray binaries, while the rest have masses $\sim 10^{6}-10^{9.5} M_{\odot}$ and are found in galactic nuclei. A variety of methods are being tried to estimate the spin parameters of the candidate black holes. There is strong circumstantial evidence that many of the objects have event horizons, so there is good reason to believe that the candidates are true black holes. Recent MHD simulations of magnetized plasma accreting on rotating black holes seem to hint that relativistic jets may be produced by a magnetic analog of the Penrose process.
\end{abstract}

Subject headings: accretion, accretion disks — active galactic nuclei — black hole physics - X-rays: binaries

\section{Introduction}

While physicists have been grappling with the theory of black holes (see the many articles in this volume), astronomers have been searching for real-life examples of black holes in the universe. This search has been remarkably successful. Dozens of excellent black hole candidates have been identified, and their properties are being investigated with a variety of techniques.

Black hole (BH) astrophysics began in the 1960s with the discovery of quasars and other active galactic nuclei (AGN) in distant galaxies. Early on it became clear that the most natural explanation for the extraordinary luminosity of AGN is the release of gravitational 
energy through accretion of gas onto supermassive BHs (see Rees 1984 for a review). BH astrophysics received a boost in the 1970s with the discovery of the X-ray binary (XRB) Cygnus X-1 (Bolton 1972). The X-ray-emitting compact star in this binary seemed to be unusually massive, so it was inferred to be a BH (e.g., Gies \& Bolton 1986). The next important breakthrough came with the work of McClintock \& Remillard (1986), who showed that the source A0620-00, a member of a class of XRBs known as X-ray novae, has a mass almost certainly greater than the maximum mass of a neutron star (NS). This important discovery opened the way to many additional $\mathrm{BH}$ candidates being found in other X-ray novae. Today about 20 excellent BH candidates are known in XRBs (see McClintock \& Remillard 2004 for a recent review). In the last decade, supermassive BHs have returned to the fore as astrophysicists have obtained impressive dynamical evidence for the presence of compact, massive, dark objects in the nuclei of nearby galaxies.

\section{Basic Concepts}

Astrophysical BHs are macroscopic objects with masses ranging from several $M_{\odot}$ (in XRBs) to $10^{6}-10^{9.5} M_{\odot}$ (in galactic nuclei), where $1 M_{\odot}=1.99 \times 10^{30} \mathrm{~kg}=$ the mass of the Sun. Being so massive, these BHs are described completely by classical general relativity. As such, each BH is characterized by just three numbers: mass $M$, spin parameter $a_{*}$, defined such that the angular momentum of the $\mathrm{BH}$ is $a_{*} G M^{2} / c$, and electric charge $Q$ (Misner, Thorne \& Wheeler 1973; Shapiro \& Teukolsky 1983; Hartle 2003). Actually, an astrophysical BH is not likely to have any significant electric charge because it will usually be rapidly neutralized by surrounding plasma. Therefore, the BH can be fully characterized by measuring just two parameters, $M$ and $a_{*}$, of which the latter is constrained to lie in the range 0 (non-spinning $\mathrm{BH}$ ) to 1 (maximally-spinning $\mathrm{BH}$ ).

Using the known equation of state of matter up to nuclear density and applying general relativity, it can be shown that the maximum mass of a compact relativistic star such as a NS is $\sim 2-3 M_{\odot}$ (Nauenberg \& Chapline 1973; Sabbadini \& Hartle 1973; Rhoades \& Ruffini 1974). This result allows the following simple and powerful technique for identifying $\mathrm{BH}$ candidates:

Find a compact (relativistic) astrophysical object and measure its mass. If the mass is greater than about $3 M_{\odot}$, then the object is very likely a $B H$.

$\S 3$ reviews the candidate BHs that have been discovered so far by this technique. What is remarkable is that in the process of identifying a $\mathrm{BH}$ candidate one has already measured $M$, one of the two parameters characterizing the object. All that is left then is to measure 
$a_{*}$ (easier said than done, as we discuss in $\S 4$ ) and we will know everything there is to know about the object!

General relativity makes very precise predictions about the properties of space-time exterior to the horizon of a stationary BH. For the general case of a spinning (uncharged) $\mathrm{BH}$, the space-time is described by the Kerr metric, which simplifies to the Schwarzschild metric when $a_{*}=0$ (Misner et al. 1973; Hartle 2003). Given the metric, one can calculate the orbits of test particles around the BH. These could, in principle, be used to interpret observations and to estimate the spin of the $\mathrm{BH}(\S 4)$.

Of all the extraordinary characteristics of a $\mathrm{BH}$ none is more remarkable than the fact that it possesses an event horizon - a one-way membrane that causally isolates the "inside" of the $\mathrm{BH}$ from the rest of the universe. Matter, radiation, energy, information, etc. can fall into the $\mathrm{BH}$ from the outside, but nothing, not even light, can get out (at least within classical physics). An important question is whether the BH candidates identifed by astronomers conform to this remarkable prediction of general relativity:

Do astrophysical BH candidates possess event horizons?

Considerable progress has been made towards answering this question, as discussed in $§ 5$.

The radius of the event horizon of a non-spinning $\mathrm{BH}$ is given by the Schwarzschild radius,

$$
R_{S}=\frac{2 G M}{c^{2}}=2.95\left(\frac{M}{M_{\odot}}\right) \mathrm{km} .
$$

The radius is smaller in the case of a spinning $\mathrm{BH}$, tending to $G M / c^{2}$ as $a_{*} \rightarrow 1$.

A most interesting property of a spinning $\mathrm{BH}$ is that it has an ergosphere (Misner et

al. 1973; Hartle 2003). This is a region external to the horizon where the dragging of inertial frames by the spinning hole is so strong that the only allowed orbits are those that corotate with the BH (as seen from an intertial frame at infinity). Penrose (1969) showed that it is possible to set up particle orbits inside the ergosphere of a spinning $\mathrm{BH}$ so as to extract rotational energy from the BH. Does the Penrose process have any application in astrophysics? This question is discussed in $\S 6$.

\section{Measuring Black Hole Mass}

The most accurate mass measurements in astrophysics are made via dynamical methods. Consider a test particle that is in a circular orbit around a mass $M$. If the orbit is wide 
enough for Newtonian physics to apply, then we have

$$
M=\frac{v^{2} r}{G}=\frac{4 \pi^{2} r^{3}}{G P_{\mathrm{orb}}^{2}}=\frac{v^{3} P_{\mathrm{orb}}}{2 \pi G},
$$

where $r$ and $P_{\text {orb }}$ are the radius and period of the orbit and $v$ is the velocity of the particle. By measuring any two of $v, r$ and $P_{\text {orb }}$, we may estimate $M$. Equation (2) is modified in a straightforward way when the orbit is non-circular. For instance, the relation $M=$ $4 \pi^{2} r^{3} / G P_{\text {orb }}^{2}$ continues to be valid, provided $r$ is taken to be the semi-major axis of the elliptical orbit of the test particle.

\subsection{X-ray Binaries}

In the case of a $\mathrm{BH} \mathrm{XRB}$, it is relatively easy to measure the period $P_{\text {orb }}$ of the orbit and the maximum line-of-sight Doppler velocity $K_{c}=v \sin i$ of the companion star to the $\mathrm{BH}$, where $i$ is the inclination angle of the binary orbit. From these, one can calculate the "mass function" $f(M)$ :

$$
f(M) \equiv \frac{K_{c}^{3} P_{\text {orb }}}{2 \pi G}=\frac{M \sin ^{3} i}{\left(1+M_{c} / M\right)^{2}},
$$

where $M$ is the mass of the $\mathrm{BH}$ candidate and $M_{c}$ is the mass of the companion star. Comparing equation (3) with equation (2) we see two differences. First, because we measure only the line-of-sight component of the orbital velocity, the mass function differs from the BH mass by a factor of $\sin ^{3} i$. Often, we have an independent estimate of $i$ from the the light curve of the binary, but this estimate is rarely as reliable as the measurements of $K_{c}$ and $P_{\text {orb }}$. The second difference is that the secondary is not a test particle but has a finite mass. This gives the factor of $\left(1+M_{c} / M\right)^{2}$ in the denominator, which corrects for the reflex motion of the BH. Therefore, one requires an independent estimate of $M_{c}$ before one can calculate $M$. In XRBs like $\mathrm{Cyg} \mathrm{X}-1, M_{c}$ is large and seriously affects our ability to estimate $M$. In contrast, systems like A0620-00 and other X-ray novae have low-mass companions which often have masses less than 10 percent of $M$. In these systems, even a very approximate estimate of $M_{c}$ is sufficient.

By combining measurements of $K_{c}$ and $P_{\text {orb }}$ with estimates of $i$ and $M_{c}$, the masses of the compact X-ray-emitting stars in a number of XRBs have been measured. Presently, 20 XRBs have been shown to have $M>3 M_{\odot}$ and these objects are all excellent $\mathrm{BH}$ candidates (McClintock \& Remillard 2004). However, the mass estimates are reliable only if $i$ (and to a lesser extent $M_{c}$ ) is well determined, which is not always the case. Fortunately, an inspection of equation (3) shows that the mass function $f(M)$, which depends only on the two accurately measured quantities $K_{c}$ and $P_{\text {orb }}$, is a strict lower bound on $M$. Most of 
the 20 XRBs have $f(M)$ itself greater than or of order $3 M_{\odot}$. Therefore, these systems are excellent $\mathrm{BH}$ candidates, regardless of uncertainties in their inclinations and companion star masses.

\subsection{Galactic Nuclei}

In the center of our Milky Way Galaxy is a dark massive object whose existence has been inferred for a number of years from its effect on the motions of stars and gas in its vicinity. Recently, high resolution infrared observations have enabled two independent groups to follow the orbits of individual stars around this object (Schödel et al. 2002; Eisenhauer et al. 2003; Ghez et al. 2003, 2004). Movie1 and Movie2 show time-elapsed images of the Galactic Center region revealing the (eccentric) orbits of several stars. By modeling the orbits with Newtonian dynamics - essentially using the generalization of equation (2) for elliptical orbits - the mass of the dark object has been estimated to be $3.7 \pm 0.2 \times 10^{6} M_{\odot}$. The mass must lie within about $2 \times 10^{13} \mathrm{~m}$ (the distance of closest approach of the observed stars), strongly suggesting that the object is a $\mathrm{BH}$. If the object is not a $\mathrm{BH}$, then the only reasonable hypothesis is that it is a dense cluster of dark compact stars. However, such a cluster would be short-lived and would become a BH in much less time than the age of the Galaxy (Maoz 1998), so this is not a very likely scenario.

A famous radio source called Sagittarius $A^{*}\left(\operatorname{Sgr} A^{*}\right)$ is located very close to the position of the dark mass described above; it has for long been suspected to be the supermassive $\mathrm{BH}$. Reid \& Brunthaler (2004) have followed the motion of Sgr A* over a period of eight years using radio interferometry and have shown that the component of its velocity perpendicular to the plane of the Galaxy is $-0.4 \pm 0.9 \mathrm{~km} \mathrm{~s}^{-1}$, consistent with zero. Since Sgr A* should experience Brownian motion as a result of gravitational interactions with stars in its vicinity, its lack of apparent motion implies that it has a large mass. Depending on the statistical method that one employs and the confidence level that one seeks, one infers that Sgr A* must have a mass of at least $10^{5} M_{\odot}$; in fact, Sgr $\mathrm{A}^{*}$ most likely encompasses the entire dark mass in the Galactic Center. The image of Sgr A* as measured in millimeter radio waves indicates an angular size of about 240 micro-arcsecond (Bower et al. 2004), which corresponds to $27 R_{S}$ for a $\mathrm{BH}$ mass of $3.7 \times 10^{6} M_{\odot}$ and a distance of $8 \mathrm{kpc}$. Thus, Sgr A* has both a large mass and a very small radius. Surely it must be a BH!

The nearby galaxy NGC 4258 is another remarkable object, with a disk of gas in its nucleus that emits radio waves via maser emission from water molecules (Miyoshi et al. 1995; Greenhill et al. 1995; Herrnstein et al. 1998). Radio interferometry measurements have shown that the gas follows circular orbits with a nearly perfect Keplerian velocity profile 
$\left(v \propto r^{-1 / 2}\right.$, see Fig. 1). Furthermore, the acceleration of the gas has been measured and it too is consistent with Keplerian dynamics (Bragg et al. 2000). From these measurements it is inferred that there is a dark object with a mass of $3.5 \times 10^{7} M_{\odot}$ confined within $\sim 4 \times 10^{15}$ $\mathrm{m}$ of the center of NGC 4258. The case for this dark mass being a $\mathrm{BH}$ is again extremely strong.

Supermassive BHs have been inferred in the nuclei of many other nearby galaxies by means of optical observations, especially with the Hubble Space Telescope (Kormendy \& Richstone 1995; Pinkney et al. 2003). One or two galaxies have coherent gas disks at the center whose orbital velocities provide information on the enclosed mass. For the rest, one measures the velocity dispersion of stars near the center of the galaxy and applies the virial theorem - essentially a statistical version of equation (2) - to infer the enclosed mass. Masses in the range $10^{6} M_{\odot}$ to $3 \times 10^{9.5} M_{\odot}$ have been estimated by this means in about 20 galaxies. Although the constraints on the radii of the dark mass concentrations in these galactic nuclei is relatively poor (compared to Sgr A* or NGC 4258), nevertheless the case for identifying the objects as supermassive BHs is strong.

Central black hole masses are difficult to measure for more distant galaxies. Techniques such as "reverberation mapping" (see Horne et al. 2004) — another application of the virial theorem - can be used if the black hole is an AGN and shows variability. Such methods are less accurate than the ones described above, but they are valuable since they provide black hole mass estimates for a large sample of galaxies.

\subsection{The Mass Distribution of Astrophysical Black Holes}

The two categories of BHs described above are clearly very distinct from each other, with very different masses: $M \sim$ few $-20 M_{\odot}$ for stellar-mass BHs in XRBs and $M \sim 10^{6}-10^{9.5} M_{\odot}$ for supermassive BHs in AGN. The BHs in XRBs are clearly the remnants of very massive stars (say with initial masses $M>30 M_{\odot}$ ) at the end of their lives. But how exactly are the $\mathrm{BHs}$ in galactic nuclei formed, and how do they evolve?

Observations have revealed interesting correlations between the supermassive BHs in the nuclei of galaxies and the properties of their host galaxies. For instance, the BH mass appears to be roughly $0.1 \%$ of the mass of the galaxy bulge (Magorrian et al. 1998; Häring \& Rix 2004), and there is an even tighter correlation between the BH mass and the velocity dispersion of the bulge (Ferrarese \& Merritt 2000; Tremaine et al. 2002); the latter quantity is, of course, a measure of the galaxy mass via the virial theorem. These correlations suggest that there is an intimate connection between the supermassive $\mathrm{BH}$ and the galaxy, even 
though the BH constitutes only a small fraction of the galaxy in terms of mass. A number of explanations have been offered for the correlations (e.g., Rees \& Silk 1998; Murray, Quataert \& Thompson 2005), but there is no real understanding of the correlation at present.

Are the two kinds of BHs discussed above the only ones in the universe or are there other kinds, e.g., intermediate mass BHs with masses of say $10^{3}-10^{4} M_{\odot}$ ? This question has attracted recent attention. In several nearby galaxies there is a class of ultra-luminous X-ray sources (Fabbiano 1989; Colbert \& Mushotzky 1999) which seem to be too bright to be ordinary $10 M_{\odot} \mathrm{BHs}$; some of these sources have luminosities of $10^{41} \mathrm{erg} \mathrm{s}^{-1}$ or more, whereas the nominal maximum steady luminosity of a gravitating object is the Eddington limit, $L_{\mathrm{Edd}}=1.3 \times 10^{39}\left(M / 10 M_{\odot}\right) \mathrm{erg} \mathrm{s}^{-1}$. The Eddington limit is the luminosity at which the outward acceleration of gas by radiation pressure is just equal to the inward acceleration by gravity. For luminosities greater than $L_{\text {Edd }}$, the radiation force is expected to overwhelm gravity and to cause the accretion rate to reduce such that the luminosity falls below $L_{\text {Edd }}$ (see Shapiro \& Teukolsky 1983 for additional discussion). Although there are ways around the Eddington limit, these require special conditions; so there is a good chance that at least some of the ultraluminous X-ray sources are much more massive than $10 M_{\odot}$. On the other hand, the sources are not likely to be (underluminous) $10^{6} M_{\odot}$ BHs because they are generally located away from the nuclei of their host galaxies. Also, in some galaxies, several ultra-luminous sources have been found whereas only one supermassive BH is typically found in a galaxy.

It is unclear at present what exactly the ultra-luminous X-ray sources are and whether they are even a single homogeneous population (see Miller \& Colbert 2004 for a review). Dynamical mass measurements would obviously settle the issue. Unfortunately, none of the sources has a confirmed binary companion, so there is no prospect of making a robust mass measurement any time soon. If ultraluminous X-ray sources are ultimately confirmed to be intermediate mass BHs, an interesting question would then need to be settled: are they a distinct new population or are they just an extended tail of the mass distribution of either stellar-mass BHs or supermassive BHs?

\section{Estimating Black Hole Spin}

Estimating the mass of a $\mathrm{BH}$ is relatively easy since mass has a measurable effect even at large radii, where Newtonian gravity applies. Spin, on the other hand, does not have any Newtonian effect - the orbit of a planet in the solar system, for instance, would be the same whether the Sun corotates or counter-rotates with the planetary orbit. Only for relativistic orbits does spin have measurable effects. Therefore, to measure $a_{*}$, we need test particles on 
orbits with very small radii. Fortunately, astrophysical BHs do have such test particles in the form of accreting gas.

When considering circular orbits in a BH spacetime (e.g., Misner et al. 1973; Shapiro \& Teukolsky 1983; Hartle 2003), a key concept is the innermost stable circular orbit, or ISCO, with a radius designated $R_{\mathrm{ISCO}}$ (also referred to as the marginally stable orbit). Circular orbits with radii $R \geq R_{\mathrm{ISCO}}$ are stable to small perturbations, whereas those with $R<R_{\mathrm{ISCO}}$ are unstable. Figure 2 shows the variation of $R_{\mathrm{ISCO}}$ with $a_{*}$. For a maximally spinning $\mathrm{BH}, R_{\mathrm{ISCO}}=G M / c^{2}$ if the orbit corotates with the $\mathrm{BH}\left(a_{*}=+1\right.$ in Fig. 2$)$ and $R_{\mathrm{ISCO}}=9 G M / c^{2}$ if it counter-rotates $\left(a_{*}=-1\right)$; for a non-spinning $\mathrm{BH}\left(a_{*}=0\right)$, $R_{\mathrm{ISCO}}=6 G M / c^{2}$. Corresponding to changes in $R_{\mathrm{ISCO}}$, there are variations in the angular velocity of an orbiting particle at $R_{\mathrm{ISCO}}$ (as measured at infinity), and in the binding energy of the particle. These variations are shown in Figure 2.

The gas in an accretion disk starts from large radii and spirals in through a sequence of nearly circular orbits as it viscously loses angular momentum. When the gas reaches the ISCO, no more stable circular orbits are available, so the gas accelerates radially and freefalls into the BH. Thus, the ISCO serves effectively as the inner edge of the accretion disk. A variety of observational methods have been proposed for estimating the radius $R_{\text {in }}=R_{\mathrm{ISCO}}$ of the disk inner edge, or one of the other quantities plotted in Figure 2, with a view to thereby estimating $a_{*}$.

\subsection{Spectral Fitting}

When a $\mathrm{BH}$ has a large mass accretion rate $\dot{M}$, corresponding to an accretion luminosity $L_{\text {acc }}$ above a few per cent of $L_{\text {Edd }}$, the accreting gas tends to be optically thick and to radiate approximately as a blackbody. In this spectral state, called the "high soft state" (McClintock \& Remillard 2004), one can theoretically calculate the flux of radiation $F(R)$ emitted by the accretion disk, and hence obtain the effective temperature profile $T_{\text {eff }}(R) \equiv[F(R) / \sigma]^{1 / 4}$, where $\sigma$ is the Stefan-Boltzmann constant. If the disk emits as a true blackbody at each radius, it is a simple matter to calculate the total spectral luminosity $L_{\nu} d \nu$. By comparing this quantity with the spectral flux $F_{\nu} d \nu$ received at Earth, one obtains an estimate of $R_{\text {in }}^{2} \cos i / D^{2}$ (essentially the projected solid angle of the disk), where $i$ is the inclination angle and $D$ is the distance to the source.

In a few BH XRBs, sufficiently reliable estimates of $i, D$ and $M$ are available to carry out this exercise and thus to estimate $R_{\text {in }} /\left(G M / c^{2}\right)$. If the inner edge of the disk is at the ISCO, as is very likely in the "high state," one then obtains $a_{*}$ (via Fig. 2). This method 
was first used by Zhang, Cui \& Chen (1997, see also Sobczak et al. 1999) for the XRBs GRO J1655-40 and GRS 1915+105, and more recently by Li et al. (2004) for the source $4 \mathrm{U} 1543-47$.

A major weakness of this method is that a number of effects - frequency-dependent opacity, Thomson/Compton scattering, etc. - will cause the spectrum of an accretion disk to deviate from a blackbody. It is traditional to model these effects by retaining the blackbody assumption but artificially increasing the temperature of the emitted radiation by a spectral hardening factor $f \sim 1.7$ (Shimura \& Takahara 1995). The problem is that the correction is very approximate. This might still be tolerable if the magnitude of the correction were small, but in fact the correction is quite large. Therefore, spin estimates obtained by this method should be treated with caution. Also, the method requires accurate estimates of $M, i$ and $D$, whereas the next two methods described below require fewer parameters to be measured.

\subsection{Quasi-Periodic Oscillations}

For several BH XRBs, the power spectrum of intensity variations shows one or two peaks (more like bumps in some cases) at frequencies of a few hundred Hz. The peaks are relatively broad, indicating that they do not correspond to coherent oscillations but rather to quasiperiodic oscillations (QPOs) with a fairly low quality factor $Q$ (McClintock \& Remillard 2004). The observed high frequency suggests that the oscillations arise in gas that is close to the BH. This gas is presumably strongly influenced by relativistic effects, including in particular effects associated with the spin of the BH. However, there is at present no clear understanding of exactly what causes the oscillations. They could be trapped vibration modes of the disk (Okazaki, Kato \& Fukue 1987; Nowak \& Wagoner 1991) or characteristic periods of test particle orbits (Stella \& Vietri 1998) or vibrations at the interface between gas and magnetic fields (Li \& Narayan 2004), or something else altogether. An important clue is that frequencies in the ratio of 3:2 are often seen, so it would appear that there is a resonance of some kind between different modes (Abramowicz \& Kluzniak 2001).

One possibility that has attracted attention is that the QPO with the highest frequency in each $\mathrm{BH} \mathrm{XRB}$ corresponds to the orbital (Keplerian) frequency of gas blobs at some characteristic radius; it is plausible that this radius corresponds to the inner edge of the disk. Since the Keplerian frequency is proportional to $1 / M$ and moreover depends on $a_{*}$ (assuming that $R_{\mathrm{in}}=R_{\mathrm{ISCO}}$, Fig. 2), one can use this method to estimate $a_{*}$ provided an estimate of $M$ is available. The method has been applied to a few BH XRBs (e.g., Strohmayer 2001). Recently, there has been tentative evidence for QPOs with a period of 17 minutes 
in the infrared emission from Sgr A*, the supermassive BH in the Galactic Center (Genzel et al. 2003). If the QPOs correspond to the Keplerian frequency at any radius $R>R_{\mathrm{ISCO}}$, then the $\mathrm{BH}$ must be spinning with $a_{*}>0.5$.

There is at present no clear evidence that the observed QPOs do correspond to the Keplerian frequency (for instance, they may be related to a precession frequency, see Cui, Zhang \& Chen 1998; Stella \& Vietri 1998). Nor is there any guarantee that the gas producing the variations is located at $R_{\mathrm{ISCO}}$. Both assumptions are certainly plausible, but it would be

nice to have corroborating evidence. On the other hand, a strength of this method is that it does not require estimates of $i$ or $D$ - all that is needed is an accurate estimate of $M$ and a physical model of the oscillations. As described in $\S 3$, mass estimates are generally quite reliable, so the future of this method depends very much on establishing a quantitative physical model of the QPOs.

\subsection{Relativistic Iron line}

Tanaka et al. (1995) discovered a strong broad spectral line in the X-ray spectrum of the AGN MCG-6-30-15. They interpreted the line as fluorescent iron $\mathrm{K} \alpha$ emission from cool gas in the accretion disk. Similar broad lines have been seen in a few other AGN and XRBs (see Reynolds \& Nowak 2003 for a review). Whereas the rest energy of the iron line is $6.4 \mathrm{keV}$, the observed line extends from about $4-7 \mathrm{keV}$, the result of broadening by Doppler blue- and red-shifts as well as gravitational redshift.

The line width - indeed the entire shape of the line - depends on several factors: (i) The radius range over which the emission occurs is clearly relevant, especially the position of the innermost radius of the disk; since $R_{\text {in }}=R_{\mathrm{ISCO}}$ depends on $a_{*}$, the $\mathrm{BH}$ spin has an important effect. (ii) The disk inclination $i$ is also important, since Doppler effects are much larger for an edge-on disk than for a face-on disk. (iii) The line emissivity as a function of $R$ strongly influences the line shape; the emissivity depends on the pattern of hard X-ray irradiation of the disk surface for which there is no reliable theory available at present.

Given a system with a broad iron line, and assuming that the radiating gas follows Keplerian orbits with radii $R \geq R_{\mathrm{ISCO}}$, one can fit the shape of the line profile by adjusting $a_{*}, i$ and the emisivity function; the latter is usually modeled as a power-law in radius, $R^{-\beta}$. Reynolds \& Nowak (2003) show how the line shape varies with $a_{*}, i$ and $\beta$. With good signal-to-noise data, it is clear that one would be able to fit all three parameters. The effect of $a_{*}$ is particularly dramatic. As the $\mathrm{BH}$ spin increases, the inner edge of the disk comes closer to the horizon (Fig. 2) and the velocity of the gas increases substantially. This gives 
a wider range of Doppler shifts, as well as a larger gravitational redshift. As a result the line extends down to very low energies below $4 \mathrm{keV}$ (especially when $\beta$ is large). The detection of such extreme levels of broadening may be taken as strong indication of a rapidly spinning $\mathrm{BH}$.

This method of estimating the BH spin has some weaknesses. First, there is no guarantee that the emissivity varies with radius as a simple power-law. Second, the method depends crucially on the assumption that the line emission cuts off abruptly inside $R_{\mathrm{ISCO}}$. However, the gas in the plunging region inside $R_{\mathrm{ISCO}}$ may also produce fluorescent emission (since it is likely to be irradiated just as much as the gas outside $\left.R_{\mathrm{ISCO}}\right)$. Thus one could have highly redshifted emission even without a rapidly spinning BH (Reynolds \& Begelman 1998). On the other hand, the method does not require any knowledge of the $\mathrm{BH}$ mass or the distance, and it solves for the disk inclination $i$ using the same line data from which $a_{*}$ is estimated. These are important advantages.

In the case of MCG-6-30-15, different observations have revealed different shapes for the line, suggesting that the line is produced by a highly variable mechanism. The data confirm that the emission comes from a relativistic disk (e.g., Vaughan \& Fabian 2004) and at least some of the datasets can be interpreted in terms of a rapidly spinning BH, e.g., assuming that there is no emission from within the ISCO, Reynolds et al. (2004b) estimate $a_{*}>0.93$. Among BH XRBs, the source GX 339-4 shows a broad iron line which seems to indicate $a_{*}>0.8$ (Miller et al. 2004). Dovciak, Karas \& Yaqoob (2004) discuss some caveats in using the iron line to estimate $a_{*}$.

Looking to the future, given a sufficiently clean system and an X-ray telescope with high sensitivity (e.g., the proposed Constellation-X and XEUS satellite missions), one might imagine not just measuring $a_{*}$ of a $\mathrm{BH}$ but even detecting higher-order effects and thereby testing general relativity. It is not known how common broad iron lines are in the X-ray spectra of AGN and XRBs - some well-studied sources show clear narrow iron lines and a puzzling absence of any broad line (Pounds et al. 2003; Reynolds et al. 2004a) — and therefore it is unclear how many favorable sources will be available for serious modeling. Also, the variability of the line with time means that it will be challenging to make fundamental tests of gravity with this method. On the other hand, the variability could provide interesting opportunities to study disk dynamics and turbulence, as Movie3 and Movie4 show. 


\subsection{Radiative Efficiency of AGN}

The radiative efficiency of an accretion disk is defined as the energy it radiates per unit accreted mass: $\eta=L_{\text {acc }} / \dot{M} c^{2}$. It is determined by the binding energy of gas at the ISCO, which depends on $a_{*}$ (Fig. 2). For a non-rotating BH, $\eta=0.057$, whereas a corotating disk around a maximally-rotating $\mathrm{BH}\left(a_{*}=1\right)$ has a much higher efficiency, $\eta=0.42$.

In a typical accretion system, one can easily measure $L_{\text {acc }}$ (provided the distance is known), but one practically never has an accurate estimate of $\dot{M}$, so one cannot calculate $\eta$ with the precision needed to estimate $a_{*}$. However, there is one situation in which it is possible. From observations of high redshift AGN, one can estimate the mean energy radiated by supermassive BHs per unit volume of the universe. Similary, by taking a census of supermassive $\mathrm{BHs}$ in nearby galaxies, one can estimate the mean mass in BHs per unit volume of the current universe. Assuming that supermassive BHs acquire most of their mass via accretion (a not unreasonable hypothesis), one can divide the two quantities to obtain the mean radiative efficiency of AGN. This is a variant of an idea originally due to Soltan (1982; see also Merritt \& Ferrarese 2001). The current data suggest an efficiency $\eta \sim 0.1-0.15$ for supermassive BHs on average (Elvis, Risaliti \& Zamorani 2002; Yu \& Tremaine 2002), and $\sim 0.2$ or even larger for the most massive systems (Yu \& Tremaine 2002). Such large values of $\eta$ are possible only if supermassive BHs have significant rotation.

There are several uncertainties in the above argument, but many of them only cause $\eta$ to be underestimated. Therefore, it does appear that supermassive BHs as a class must have substantial rotation. However, it should be noted that this is only a statistical result for the population as a whole, and the method does not say anything about the rotation of any specific $\mathrm{BH}$.

\section{Evidence for the Event Horizon}

While the measurements of mass and spin described in the previous sections are very impressive, it is important to keep in mind that there is as yet no definite proof that any of the $\mathrm{BH}$ candidates discovered so far is truly a $\mathrm{BH}$. All we know is that the objects are too massive $\left(>3 M_{\odot}\right)$ to be NSs. But mass measurements by themselves cannot prove that the objects are BHs.

To prove that an object is a $\mathrm{BH}$ requires an unambiguous demonstration that it possesses an event horizon, a challenging (perhaps technically impossible, Abramowicz, Kluzniak \& Lasota 2002) undertaking. Nevertheless, considerable progress has been made, and there is strong circumstantial evidence that many of the $\mathrm{BH}$ candidates discovered so far do possess 
event horizons.

\subsection{Luminosities of Quiescent BHs}

When a $\mathrm{BH}$ accretes at a rate much less than the Eddington rate, the radiative efficiency of the accreting gas is found to be very small, i.e., the efficiency parameter $\eta=L_{\text {acc }} / \dot{M} c^{2}$ defined earlier is found to be $\ll 1$. The best-known example is $\mathrm{Sgr} \mathrm{A}^{*}$, which is extremely underluminous, $L_{\text {acc }} \sim 10^{36} \mathrm{erg} \mathrm{s}^{-1}$, compared to the Eddington luminosity $L_{\text {Edd }} \sim 5 \times$ $10^{44} \mathrm{erg} \mathrm{s}^{-1}$ for its mass. What is interesting is that there is considerable gas available around Sgr $\mathrm{A}^{*}$, and if the accretion flow possessed a standard efficiency $\eta \sim 0.06-0.4$, the luminosity would be orders of magnitude larger than that observed.

Narayan, Yi \& Mahadevan (1995) came up with an explanation for the unusually low luminosity. Building on previous work by Narayan \& Yi (1994, 1995ab) and Abramowicz et al. (1995), they suggested that Sgr A* may be accreting via an advection-dominated accretion flow (ADAF). The key feature of an ADAF is that the gravitational energy that is released as gas sinks in the potential well of the $\mathrm{BH}$ remains locked up in the gas as thermal energy (or entropy) instead of being radiated. This can happen if the gas has a low enough density, as is expected at low mass accretion rates $\dot{M}$, so that the charged particles in the gas rarely scatter off one another and therefore do not radiate very much. The result is that the gas accretes down to the center with a very high temperature $\left(\sim 10^{12} \mathrm{~K}\right)$. When the gas reaches the $\mathrm{BH}$ horizon, it simply falls in with all its thermal energy, and the accretion flow is extremely dim.

The above explanation for Sgr A*'s unusually low luminosity obviously requires the source to have an event horizon. If Sgr A* were to have a surface, then the accreting hot gas would come to a stop when it hits the surface and would radiate all its stored thermal energy (since the density would go up by a large factor and the radiative efficiency would increase correspondingly). Therefore, to understand the extraordinary dimness of Sgr A*, we require the object to be a true BH with an event horizon (Narayan et al. 1998). Note that Sgr A* is not an isolated example. The vast majority of supermassive BHs in nearby galaxies are very underluminous relative to the gas supply available to them (Fabian \& Canizares 1988). The same explanation, viz., an ADAF accreting onto an event horizon, will work for all these objects (Fabian \& Rees 1995).

One major complication with this argument is that the gas in an ADAF is very weakly bound to the $\mathrm{BH}$ because of its large temperature. It therefore tends to be ejected from the system rather easily (Narayan \& Yi 1994, 1995a; Blandford \& Begelman 1999; Quataert 
\& Narayan 1999). As a result, less gas reaches the BH than one might naively expect given the amount of gas supply, and so the luminosity deficit is less serious than one might have originally thought. Quantitatively, of the luminosity deficit of $\sim 10^{-4}$ seen in $\operatorname{Sgr} A^{*}$, roughly $10^{-2}$ is from gas ejection and the remaining $\sim 10^{-2}$ is the result of advection and the disappearance of energy through the event horizon. The argument for the event horizon thus survives, but it is weakened a little.

What one would really like to do is compare ADAFs around two accreting objects, one with a surface and one with a suspected event horizon. In such a comparison, if the object with a surface is much brighter than the other object, then the case for an event horizon in the latter would be very strong. Narayan, Garcia \& McClintock (1997) suggested that precisely such a comparison could be done using XRBs.

As mentioned in $\S 1$, most of the known stellar-mass $\mathrm{BH}$ candidates are in a class of XRBs called X-ray novae. These systems are characterized by a variable mass accretion rate, and tend to spend most of their time in a quiescent state with a very low $\dot{M}$ and $L_{\text {acc }}$. Only occasionally do they go into outburst, when they accrete with high $\dot{M}$ and become bright. Spectral observations of quiescent BH XRBs can be explained in terms of an ADAF (see Narayan, Mahadevan \& Quataert 1998 for a review). Interestingly, in addition to the many X-ray novae that have been identified with $\mathrm{BH}$ candidates, there are other X-ray novae in which the accreting object is a NS. These latter systems too go into long periods of quiescence, when they presumably have ADAFs. Narayan et al. (1997) suggested that by comparing the quiescent luminosities of BH and NS X-ray novae, one could test for the presence of event horizons in the former: the $\mathrm{BH}$ candidates should be much dimmer than the NSs. The very limited data available in 1997 seemed to bear this prediction out.

The observational situation has improved considerably since 1997 (see Garcia et al. 2001; Hameury et al. 2003; McClintock et al. 2004), and one can now state with considerable confidence that quiescent BH X-ray novae are very much dimmer than quiescent NS X-ray novae. The luminosity difference is a factor of 1000 when measured in Eddington units, and a factor of 100 when compared directly without Eddington scaling. Figure 3 shows the latest data on quiescent luminosities plotted against the binary orbital period $P_{\text {orb }}$. The mass accretion rate is determined largely by the latter quantity (Lasota \& Hameury 1998; Menou et al. 1999) and hence NS X-ray novae and BH X-ray novae with similar orbital periods are likely to have similar mass accretion rates. The fact that their luminosities are so different is then both surprising and significant, and must reflect some fundamental difference between the two kinds of object. While a few counter-explanations have been proposed (see Narayan, Garcia \& McClintock 2002; Hameury et al. 2003; McClintock et al. 2004 for references and rebuttals), the most natural explanation of the observations is that accretion in quiescent 
XRBs occurs via an ADAF; the $\mathrm{BH}$ candidates are dimmer because they have event horizons through which they swallow all the advected energy. In other words, black holes really are black relative to other compact objects with surfaces!

A related argument is based on the X-ray spectra of quiescent X-ray novae. Most quiescent NSs have X-ray spectra consisting of two distinct components: a power-law component and a thermal blackbody-like component. The former is identified with a hot (likely advection-dominated) accretion flow, and the latter with emission from the surface of the NS. McClintock et al. (2004) analyzed the spectrum of the BH X-ray nova XTE J1118+480 in quiescence and established a very tight limit on a thermal component: $L_{\text {th }}<9.4 \times 10^{30} \mathrm{erg} \mathrm{s}^{-1}$ at the $99 \%$ confidence level. (There is a very clear power-law component in the spectrum, as one would expect for a hot ADAF.) In comparison, the thermal component in NS systems is typically much brighter, $\sim$ few $\times 10^{32}$ to few $\times 10^{33} \mathrm{erg} \mathrm{s}^{-1}$. Since the thermal component in NSs is identified with surface emission, the lack of a similar component in XTE J1118+480 argues for the lack of a surface in this source, i.e., the object must have an event horizon. This independent argument based on the spectrum bolsters the previous discussion, which was based purely on luminosity.

\subsection{Absence of a Boundary Layer}

When an accretion disk, especially one with relatively cool gas in the "high soft state," is present around a compact star, a narrow viscous boundary layer will form at the radius where the rapidly orbiting gas meets the surface of the star. A considerable amount of heat energy is expected to be liberated in this boundary layer. No boundary layer is expected if the central object is a black hole.

Sunyaev \& Revnivtsev (2000) showed that the variability power spectra of NS XRBs have significant power even at frequencies as high as $1 \mathrm{kHz}$, whereas the power spectra of $\mathrm{BH}$ XRBs decline strongly above about $50 \mathrm{~Hz}$. Part of the difference is explained by the mass difference between the two objects, since characteristic dynamical frequencies scale as $M^{-1}$. But even after allowing for this effect, there is still a residual difference in the cutoff frequencies. Sunyaev \& Revnivtsev suggested that NSs have an additional high frequency component in their variability spectra coming from the boundary layer region, whereas the $\mathrm{BH}$ candidates lack this component. If correct, this argument implies that $\mathrm{BH}$ candidates must have event horizons.

In related work, Done \& Gierlinsky (2003) studied the X-ray spectra of bright BH XRBs

and NS XRBs. They defined a "hard color" and a "soft color" to characterize the observed 
spectra and plotted these quantitites for BH and NS systems in a color-color diagram. They showed that there are several similarities between BHs and NSs, but also some clear differences. In particular, a certain kind of pattern in the color-color diagram is seen only from NS systems. Done \& Gierlinsky interpreted this unique spectral component as emission from a boundary layer. If their identification is correct, then the lack of a similar component in $\mathrm{BH}$ XRBs implies that a boundary layer is missing, i.e., these objects must have event horizons.

\subsection{Type I X-ray Bursts}

When gas accretes on the surface of a neutron star, it becomes denser and hotter as it sinks under the weight of continued accretion, until at a certain depth it ignites thermonuclear reactions. The ignited reactions are usually unstable, causing the accreted layer of gas to burn explosively within a very short time. After the fuel is consumed, the star reverts to its accretion phase until the next thermonuclear instability is triggered. The star thus undergoes a semi-regular series of thermonuclear explosions (see Lewin, van Paradijs \& Taam 1993; Bildsten 1998; for reviews). These thermonuclear bursts, called Type I bursts, were first discovered from X-ray binaries by Grindlay et al. (1976).

In a typical Type I X-ray burst, the luminosity of the neutron star increases to nearly the Eddington limit in less than a second, and the flux then declines over a period of seconds to tens of seconds. The time interval between bursts is usually several hours to perhaps a day or two. The physics of Type I bursts has been widely studied, and the broad features of the phenomenon are understood. Theoretical models generally agree quite well with observations (Lewin et al. 1993; Bildsten 1998; Narayan \& Heyl 2003).

Remarkably, no Type I burst has ever been seen in any BH XRB (e.g., Tournear et al. 2003). Why should this be the case? Calculations indicate that if a BH candidate with (say) a mass of $10 M_{\odot}$ were to have a surface, it would exhibit Type I bursts with a burst rate only a factor $\sim 3$ smaller than for a NS (Narayan \& Heyl 2002; Yuan, Narayan \& Rees 2004). Yet, although thousands of bursts have been observed from NSs, not one burst has been seen from any $\mathrm{BH}$ candidate!

Narayan \& Heyl (2002) argued that the lack of bursts is strong evidence for the presence of horizons in $\mathrm{BH}$ candidates. Clearly, if the object has no surface, then gas cannot accumulate and cannot develop a thermonuclear instability. But is the lack of a surface the only reason why $\mathrm{BH}$ candidates do not have bursts? Is there any reasonable scenario in which the objects might have surfaces and still not burst? There has been some discussion of this point (see Narayan 2003; Yuan et al. 2004; also Abramowicz, Kluzniak \& Lasota 
2002). In brief, it appears that one needs to invoke very unusual physics if one wishes to explain how BH candidates could have surfaces and yet not produce Type I bursts. In fact, the requirements are so extreme that it is far more economical simply to accept that $\mathrm{BH}$ candidates have event horizons!

\subsection{Direct Imaging}

Although none of the arguments described above is absolutely rigorous, in combination they leave almost no room for any model of $\mathrm{BH}$ candidates that does not include an event horizon. Thus, it is fair to say that there is a compelling case for the presence of event horizons in astrophysical BHs. The evidence is however indirect, and one wonders whether it is possible to obtain more direct evidence.

The most promising idea is to obtain an image of the region near the event horizon of an accreting BH. At first sight this seems impossible, considering how compact astrophysical BHs are and how far away they are from us. However, the situation is not entirely hopeless. Consider for concreteness a non-rotating $\mathrm{BH}$ with a horizon at radius $R_{S}$. Because of strong light-bending in the vicinity of the $\mathrm{BH}$, a distant observer will see an apparent boundary of the $\mathrm{BH}$ at a radius of $(27)^{1 / 2} R_{S} / 2$ (Falcke, Melia \& Agol 2000). Rays with impact parameters inside this boundary intersect the horizon, while rays outside the boundary miss the horizon. The angular size of the boundary is

$$
\theta_{b}=\frac{(27)^{1 / 2} R_{S}}{2 D}=5 \times 10^{-8}\left(\frac{M}{1 M_{\odot}}\right)\left(\frac{1 \mathrm{kpc}}{D}\right) \text { mas },
$$

where mas $=$ milliarcsecond $=4.85 \times 10^{-9}$ radian. A $10 M_{\odot} \mathrm{BH}$ candidate at a distance of $1 \mathrm{kpc}$ will have $\theta_{b} \sim 10^{-6}$ mas, which is much too small to be resolved with any technique in the foreseeable future. However, Sgr A* with $M \sim 4 \times 10^{6} M_{\odot}$ and at a distance of $8 \mathrm{kpc}$ should have an angular size of $\sim 0.02$ mas which is not beyond reach. The supermassive BH in the nucleus of the nearby $(15 \mathrm{Mpc})$ giant elliptical galaxy M87, with a mass of $3 \times 10^{9} M_{\odot}$ and an expected angular size of $\sim 0.01$ mas, is another object of interest.

The best angular resolution achievable today is with radio interferometry, where angles less than 1 mas are routinely resolved. In the not too distant future, it should be possible to operate interferometers at wavelengths $\lambda<1 \mathrm{~mm}$ and with baselines as large as the diameter of the earth $b \sim 10^{4} \mathrm{~km}$. The nominal angular resolution of such an interferometer would be $\lambda / b \sim 0.02$ mas, i.e., suitable for resolving the image of Sgr $A^{*}$. Interestingly, Sgr A* radiates most of its (very small) luminosity in sub-millimeter waves, so there will be a measurable signal at these wavelengths. Moreover, there is a reasonable chance that the accreting gas 
will be optically thin so that one will be able to see through it. In addition, since the gas is advection-dominated it is likely to be quasi-spherical rather than disk-like, thus enabling us to image all around the BH.

Falcke et al. (2000) suggested that by imaging Sgr A* with sub-millimeter radio interferometry, one could check whether there is a reduction in the surface brightness inside $\theta_{b}$. They call this the "shadow of the black hole." The detection of this shadow would certainly confirm a key prediction of general relativity. But will it constitute proof of the event horizon? That is not obvious. The shadow of the BH is caused by strong gravitational bending of light rays; in particular, it reflects the existence of circular photon orbits, which is an effect of strong gravity. However, even a NS, if it were sufficiently compact, would have photon orbits. Therefore, if a shadow is seen in sub-millimeter waves in Sgr A*, all that it means is that there is no significant radiation coming from the surface of the $\mathrm{BH}$ at these wavelengths. This could be because the BH has no surface, i.e., it has an event horizon, or it could simply be that the object has a surface that happens not to radiate in this band.

Looking further into the future, there are ambitious plans to develop X-ray interferometry in space (e.g., the MAXIM mission concept, Cash 2002) to study accretion flows around supermassive black holes in other galactic nuclei. If these plans come to fruition, one could imagine mapping disks in say the X-ray iron line and watching the image change with time (e.g., Movie3 and Movie4)! Such observations are probably at least a few decades away.

\section{Extracting Energy from Spinning Black Holes}

Astrophysical BHs are expected to have non-zero angular momentum. The progenitors of stellar-mass BHs almost certainly were spinning when they collapsed, so their end-products should be spinning. Supermassive BHs too should have considerable angular momentum since they are believed to have acquired most of their mass from an orbiting accretion disk. A spinning BH has free energy associated with its rotation which can in principle be tapped, e.g., by the Penrose process. Given all this, it has been suspected for long that some of the most energetic phenomena associated with astrophysical BHs may somehow be related to $\mathrm{BH}$ spin. However, convincing evidence has been hard to come by.

Model fits of the iron line in some sources require a very steep emissivity function (see Reynolds \& Nowak 2003); most of the energy has to be emitted closer to the BH than one expects if the energy source is the accretion flow. This has led to the suggestion that perhaps a substantial fraction of the line energy is supplied by the $\mathrm{BH}$, although there is as yet no convincing model showing how this happens. 
Another promising possibility is that relativistic jets, which are so ubiquitous in accreting BHs, are launched from the ergospheres of rotating BHs. Jets with very large bulk Lorentz factors $\gamma \sim 10$ have been known for long in radio-loud AGN (Zensus 1997). More recently, moderately large Lorentz factors $\gamma \sim$ few have been seen in BH XRBs (Mirabel \& Rodríguez 1999). In addition, there is clear evidence for jets with $\gamma \sim 100$ in the ejecta of gamma-ray bursts (GRBs, Mészáros 2002). GRBs are known to be associated with the deaths of massive stars in "hypernovae," and while there is no certainty that these explosions produce BHs at their centers, there is a good chance that they do.

The original idea of Penrose for extracting energy from a rotating BH involves a particle that enters the ergosphere and then splits into two. One of the two particles falls into the BH on a negative-energy orbit, causing the mass, energy and angular momentum of the $\mathrm{BH}$ to decrease, while the other particle escapes to infinity carrying the excess energy and angular momentum with it. This process requires particular conditions (Bardeen, Press \& Teukolsky 1972) that are probably not common in nature. Another process to extract energy from a rotating $\mathrm{BH}$ is via "super-radiance" in which a a suitably tuned wave impinges on the BH and is reflected with increased energy (Press \& Teukolsky 1972). This mechanism has been suggested as an energy source for GRBs (van Putten 1999; Aguirre 2000). Presently, the most promising idea for extracting energy from a rotating BH is via magnetic fields (Znajek 1976; Blandford \& Znajek 1977) since fairly strong magnetic fields are likely to be present in all accretion flows. Magnetic fields are capable of connecting regions very close to the BH to regions farther out, thereby introducing considerable long-range coherence.

How exactly does the magnetic field tap the energy of a rotating BH? Recent general relativistic numerical MHD simulations are beginning to provide an answer. Koide et al. (2002) and Koide (2004) describe simulations of a magnetized plasma in the ergosphere of a spinning BH. They find that, near the equatorial plane, the field lines are azimuthally twisted because of the dragging of intertial frames by the spinning hole. The twist then propagates outward and transports energy along twin jets that form naturally. The results are in general agreement with an earlier proposal made by Punsly \& Coroniti (1990). In addition, Koide et al. (2002) have shown that the energy extraction mechanism is related to the Penrose process. As the field lines in the equatorial region are pulled forward, the local plasma on the field line acquires a negative energy. This plasma falls into the $\mathrm{BH}$, while a torsional Alfven wave is driven outward along the field lines, generating an outward Poynting flux and at the same time increasing the energy of plasma outside the ergosphere. The authors call it the MHD Penrose process since there is a close analogy between this mechanism and Penrose's original idea. The main difference is that the negative energy particles close to the $\mathrm{BH}$ and the positive energy particles farther out communicate via magnetic field lines, which act as energy conduits. 
Semenov, Dyadechkin \& Punsly (2004) have recently used a simplified set of equations to follow the evolution of individual magnetic flux tubes in the vicinity of a rotating $\mathrm{BH}$. Movie5 and Movie6 from their work show how a rotating BH with a magnetized plasma around it naturally develops a configuration with infalling negative energy plasma in the equatorial plane and outgoing coiled magnetic fields that carry away energy in twin jets. Much more work is obviously needed, but it appears that the connection between rotating BHs and jets is beginning to be understood.

Note that the field topology in the above-cited work is different from the traditional picture in which field lines connect the $\mathrm{BH}$ horizon to the accretion disk. The traditional field configuration seems to produce evacuated funnels with outflowing funnel walls rather than bona fide jets (de Villiers, Hawley \& Krolik 2003; McKinney \& Gammie 2004). The simulations of Koide et al. (2002) and Semenov et al. (2004) start out with field lines going from the ergosphere to infinity along the spin axis. Such a field configuration can be achieved naturally as a result of magnetized plasma falling into the BH (Narayan, Igumenshchev \& Abramowicz 2003). In fact, not only does this field configuration enable energy extraction from a rotating hole, it may also enhance the energy extracted from gas accreting onto a non-rotating hole, i.e., it can give a larger value of $\eta$ than the standard value of 0.057 shown in Fig. 2 (Narayan et al. 2003).

\section{Conclusion}

Astrophysicists are contributing in important ways to the study of BHs. They are discovering increasing numbers of $\mathrm{BH}$ candidates, they are measuring the fundamental parameters of the BHs, they are finding strong evidence that $\mathrm{BH}$ candidates have event horizons, and they are beginning to carry out additional tests of relativity in the regime of strong gravity. However, all the work done so far relates only to the classical regime. Quantum effects have remained out of reach.

The most famous quantum effect is, of course, Hawking (1974) radiation. Unfortunately, for massive BHs such as the ones discovered so far, the Hawking temperature is so small $\left[\sim 10^{-7}\left(M_{\odot} / M\right) \mathrm{K}\right]$ that there is no hope of ever detecting this radiation. The story is different in the case of BHs with $M<10^{15} \mathrm{~g}$. Such BHs will evaporate by the Hawking mechanism in a time less than the age of the universe and will explode with final luminosities $\sim 10^{20} \mathrm{erg} \mathrm{s}^{-1}$ (Shapiro \& Teukolsky 1983). If the universe made such mini-BHs in sufficient numbers, we might expect to see a few of them die in fleeting bursts of high energy emission. Given our present understanding of the formation of compact objects by stellar collapse, and given the constraints on density fluctuations in the early universe as determined from 
observations of the cosmic microwave background, there does not seem to be much chance

of finding $10^{15} \mathrm{~g}$ BHs in the universe today. However, nature has a long history of surprising us - nowhere more true than in astrophysics — so perhaps we should not give up hope altogether.

This work was supported in part by NASA grant NAG5-10780 and NSF grant AST 0307433 .

\section{REFERENCES}

Abramowicz, M. A., Chen, X., Kato, S., Lasota, J.-P., \& Regev, O. 1995, ApJ, 438, L37

Abramowicz, M. A., \& Kluzniak, W. 2001, A\&A, 374, L19

Abramowicz, M. A., Kluzniak, W., \& Lasota, J.-P. 2002, A\&A, 396, L31

Aguirre, A. N. 2000, ApJ, 529, L9

Armitage, P. J., \& Reynolds, C. S. 2003, MNRAS, 341, 1041

Bardeen, J. M., Press, W. H., \& Teukolsky, S. A. 1972, ApJ, 178, 347

Bildsten, L. 1998, in The Many Faces of Neutron Stars, eds. A. Alpar, L. Buccheri, J. van Paradijs, Dordrecht: Kluwer, p419

Blandford, R. D., \& Begelman, M. C. 1999, MNRAS, 303, L1

Blandford, R. D., \& Znajek, R. 1977, MNRAS, 179, 433

Bolton, C. T. 1972, Nature, 235, 271

Bower, G. C., Falcke, H., Herrnstein, R. M., Zhao, J.-H., Goss, W. M., \& Backer, D. C. 2004, Science, 304, 704

Bragg, A. E., Greenhill, L., Moran, J. M., \& Henkel, C. 2000, ApJ, 535, 73

Cash, W. 2002, 34th COSPAR Scientific Assembly, The Second World Space Congress, p1524

Colbert, E. J. M., \& Mushotzky, R. F. 1999, ApJ, 519, 89

Cui, W., Zhang, S. N., \& Chen, W. 1998, ApJ, 492, L53

de Villiers, J.-P., Hawley, J. F., \& Krolik, J. H. 2003, ApJ, 599, 1238 
Done, C., \& Gierlinski, M. 2003, MNRAS, 342, 1041

Dovciak, M., Karas, V., \& Yaqoob, T. 2004, ApJS, 153, 205

Eisenhauer, F., Schödel, R., Genzel, R., Ott, T., Tecza, M., Abuter, R., et al. 2003, ApJ, 597, L121

Elvis, M., Risaliti, G., \& Zamorani, G. 2002, ApJ, 565, L75

Fabbiano, G. 1989, ARA\&A, 27, 87

Fabian, A. C., \& Canizares, C. R. 1988, Nature, 333, 829

Fabian, A. C., \& Rees, M. J. 1995, MNRAS, 277, L55

Falcke, H., Melia, F., \& Agol, E. 2000, ApJ, 528, L13

Ferrarese, D., \& Merritt, D. 2000, ApJ, 539, L9

Garcia, M. R., McClintock, J. E., Narayan, R., Callanan, P., Barret, D., \& Murray, S. S. 2001, ApJ, 553, L47

Genzel, R., Schödel, R., Ott, T., Eckart, A., Alexander, T., Lacombe, F., et al. 2003, Nature, 425,934

Ghez, A. M., Duchêne, G., Matthews, K., Hornstein, S. D., Tanner, A., Larkin, J., et al. 2003, ApJ, 586, L127

Ghez, A. M., Salim, S., Hornstein, S. D., Tanner, A., Morris, M., Becklin, E. E., \& Duchene, G. 2004, ApJ, in press (astro-ph/0306130)

Gies, D. R., \& Bolton, C. T. 1986, ApJ, 304, 371

Greenhill, L. J., Jiang, D. R., Moran, J. M., Reid, M. J., Lo, K. Y., \& Claussen, M. J. 1995, ApJ, 440, 619

Grindlay, J., Gursky, H., Schnopper, H., Parsignault, D. R., Heise, J., Brinkman, A. C., \& Schrijver, J. 1976, ApJ, 205, L127

Häring, N., \& Rix, H.-W. 2004, ApJ, 604, L89

Hameury, J.-M., Barret, D., Lasota, J.-P., McClintock, J. E., Menou, K., Motch, C., et al. 2003, A\&A, 399, 631 
Hartle, J. B. 2003, Gravity: An Introduction to Einstein's General Relativity, AddisonWesley: San Francisco

Hawking, S. W. 1974, Nature, 248, 30

Herrnstein, J. R., Greenhill, L. J., Moran, J. M., Diamond, P. J., Inoue, M., Nakai, N., \& Miyoshi, M. 1998, ApJ, 497, L69

Koide, S. 2004, ApJ, 606, L45

Koide, S., Shibata, K., Kudoh, T., \& Meier, D. L. 2002, Science, 295, 1688

Kormendy, J., \& Richstone, D. 1995, ARA\&A, 33, 581

Lasota, J.-P., \& Hameury, J.-M., AIP Conf. Proc. 431, 351

Lewin, W. H. G., van Paradijs, J., \& Taam, R. E. 1993, Space Sci. Rev., 62, 223

Li, L.-X., \& Narayan, R. 2004, ApJ, 601, 414

Li, L.-X., Zimmerman, E. R., Narayan, R., \& McClintock, J. E. 2004, ApJ, submitted

Magorrian, J., Tremaine, S., Richstone, D., Bender, R., Bower, G., Dressler, A., et al. 1998, AJ, 115, 2285

Maoz, E. 1998, ApJ, 494, L181

McClintock, J. E., Narayan, R., \& Rybicki, G. B. 2004, ApJ, in press (astro-ph/0403251)

McClintock, J. E., \& Remillard, R. A. 1986, ApJ, 308, 110

McClintock, J. E., \& Remillard, R. A. 2004, in Compact Stellar X-ray Sources, eds. W.H.G. Lewin and M. van der Klis, Cambridge Univ. Press (astro-ph/0306213)

McKinney, J. C., \& Gammie, C. F. 2004, ApJ, 611, 977

Menou, K., Esin, A. A., Narayan, R., Garcia, M. R., Lasota, J.-P., \& McClintock, J. E. 1999, ApJ, 520, 276

Merritt, D., \& Ferrarese, L. 2001, MNRAS, 320, L30

Mészáros, P. 2002, ARA\&A, 40, 137

Miller, C. M., \& Colbert, E. J. M. 2004, International J. Modern Phys. D, 13, 1 
Miller, J. M., Fabian, A. C., Reynolds, C. S., Nowak, M. A., Homan, J., Freyberg, M. J., et al. 2004, ApJ, 606, L131

Mirabel, I. F., \& Rodríguez, L. F. 1999, ARA\&A, 37, 409

Misner, C. W., Thorne, K. S., \& Wheeler, J. A. 1973, Gravitation, Freeman: San Francisco

Miyoshi, M., Moran, J., Herrnstein, J., Greenhill, L., Nakai, N., Diamond, P., \& Inoue, M. 1995, Nature, 373, 127

Murray, N., Quataert, E., \& Thompson, T. A. 2005, ApJ, in press (astro-ph/0406070)

Narayan, R. 2003, Astron. \& Geophys., 44, 6.22

Narayan, R., Garcia, M. R., \& McClintock, J. E. 1997, ApJ, 478, L79

Narayan, R., Garcia, M. R., \& McClintock, J. E. 2002, in Proc. Ninth Marcel Grossmann Meeting, eds. V. G. Gurzadyan, R. T. Jantzen, R. Ruffini, World Scientific Publ., $\mathrm{p} 405$

Narayan, R., \& Heyl, J. S. 2002, ApJ, 574, L139

Narayan, R., \& Heyl, J. S. 2003, ApJ, 599, 419

Narayan, R., Igumenshchev, I. V., \& Abramowicz, M. A. 2003, PASJ, 55, L69

Narayan, R., Mahadevan, R., Grindlay, J. E., Popham, R. G., \& Gammie, C. 1998, ApJ, 492,554

Narayan, R., Mahadevan, R., \& Quataert, E. 1998, in Theory of Black Hole Accretion Disks, eds. M. A. Abramowicz, F. Bjornsson, J. E. Pringle, Cambridge Univ. Press, p148

Narayan, R., \& Yi, I. 1994, ApJ, 428, L13

Narayan, R., \& Yi, I. 1995a, ApJ, 444, 231

Narayan, R., \& Yi, I. 1995b, ApJ, 452, 710

Narayan, R., Yi, I., \& Mahadevan, R. 1995, Nature, 374, 623

Nauenberg, M., \& Chapline, G. J. 1973, ApJ, 179, 277

Nowak, M. A., \& Wagoner, R. V. 1991, ApJ, 378, 656

Okazaki, A. T., Kato, S., \& Fukue, J. 1987, PASJ, 39, 457 
Penrose, R. 1969, Riv. Nuovo Cim., 1, 252

Pinkney, J., Gebhardt, K., Bender, R., Bower, G., Dressler, A., Faber, S. M., et al. 2003, ApJ, 596, 903

Pounds, K. A., Reeves, J. N., Page, K. L., Edelson, R., Matt, G., \& Perola, G. C. 2003, MNRAS, 341, 953

Press, W. H., \& Teukolsky, S. A. 1972, Nature, 238, 211

Punsly, B., \& Coroniti, F. V. 1990, ApJ, 354, 583

Quataert, E., \& Narayan, R. 1999, ApJ, 520, 298

Rees, M. J. 1984, ARA\&A, 22,471

Reid, M. J., \& Brunthaler , A. 2004, ApJ, in press (astro-ph/0408107)

Reynolds, C. S., \& Begelman, M. C. 1998, ApJ, 488, 109

Reynolds, C. S., Brenneman, L. W., Wilms, Jörn, \& Kaiser, M. E. 2004a, MNRAS, 352, 205

Reynolds, C. S., Brenneman, L. W., \& Garofalo, D. 2004, in "From X-ray Binaries to Quasars: Black Hole Accretion on All Mass Scales, eds. T. Maccarone, R. Fender, L. Ho, Kluwer, in press (astro-ph/0410116)

Reynolds, C. S., \& Nowak, M. A. 2003, Phys. Repts., 377, 389

Rhoades, C. E., \& Ruffini, R. 1974, Phys. Rev. Lett., 32, 324

Sabbadini, A. G., \& Hartle, J. B. 1973, Ap. Space Sci., 25, 117

Schödel, R., Ott, T., Genzel, R., Hoffmann, R., Lehnert, M., Eckart, A., et al. 2002, Nature, 419,694

Semenov, V., Dyadechkin, S., \& Punsly, B. 2004, Science, 305, 978

Shapiro, S. L., \& Teukolsky, S. A. 1983, Black Holes, White Dwarfs, and Neutron Stars, Wiley: New York

Shimura, T., \& Takahara, F. 1995, ApJ, 445, 780

Silk, J., \& Rees, M. J. 1998, A\&A, 331, L1

Sobczak, G. J., McClintock, J. E., Remillard, R. A., Bailyn, C. D., \& Orosz, J. A. 1999, ApJ, 520, 776 
Soltan, A. 1982, MNRAS, 200, 115

Stella, L., \& Vietri, M. 1998, ApJ, 492, L59

Strohmayer, T. E. 2001, ApJ, 552, L49

Sunyaev, R., \& Revnivtsev, M. 2000, A\&A, 358, 617

Tanaka, Y., Nandra, K., Fabian, A. C., Inoue, H., Otani, C., Dotani, T., et al. 1995, Nature, 375,659

Tournear, D., Raffauf, E., Bloom, E. D., Focke, W., Giebels, B., Godfrey, G., et al. 2003, ApJ, 595, 1058

Tremaine, S., Gebhardt, K., Bender, R., Bower, G., Dressler, A., et al. 2002, ApJ, 574, 740

Vaughan, S., \& Fabian, A. C. 2004, MNRAS, 348, 1415

van Putten, M. H. P. M. 1999, Science, 284, 115

Yu, Q., \& Tremaine, S. 2002, MNRAS, 335, 965

Yuan, Y.-F., Narayan, R., \& Rees, M. J. 2004, ApJ, 606, 1112

Zensus, J. A. 1997, ARA\&A, 35, 607

Zhang, S. N., Cui, W., \& Chen, W. 1997, ApJ, 482, L155

Znajek, R. 1976, Nature, 262, 270 


\section{Movie Captions}

Movie1.- Shows the orbits of individual stars near the Galactic Center as measured with high resolution infrared observations. The movie runs from 1992, the initial date of the observations, to the present, and is extrapolated a few years into the future. Time is shown at top left. The star whose track (with error bars) traces a complete ellipse was fitted by Schödel et al. (2002) to a highly elliptical Keplerian orbit. From the fit they calculated the mass of the supermassive $\mathrm{BH}$ to be $3.7 \pm 1.5 \times 10^{6} M_{\odot}$. The inferred position of the $\mathrm{BH}$ is shown by the red cross. (Movie courtesy Reinhard Genzel)

Movie2.- Shows the orbits of individual stars near the Galactic Center as measured with high resolution infrared observations. The movie runs from 1995, the initial date of the observations, to the present, and is extrapolated a few years into the future. Time is shown at top left. By combining the stellar positions with Doppler radial velocity measurements and fitting to Keplerian orbits, Ghez et al. (2004) refined the mass estimate of the supermassive $\mathrm{BH}$ to $3.7 \pm 0.2 \times 10^{6} M_{\odot}$. The inferred position of the $\mathrm{BH}$ is shown by the stationary ${ }^{*}$ at the center. (Movie courtesy Andrea Ghez)

Movie3.- Shows the simulated image of a turbulent accretion disk heated by the dissipation of magnetic fields. The dark region at the center is the inner boundary of the simulation, which is at a radius of $2 R_{S}$. During the sequence the view changes from almost face-on $\left(i=1^{\circ}\right)$ to nearly edge-on $\left(i=80^{\circ}\right)$, as indicated by the bar on the right $\left(0^{\circ}\right.$ is at the bottom of the bar and $90^{\circ}$ at the top). As the inclination increases, note how the emission becomes enhanced to the left of the $\mathrm{BH}$ because of Doppler boost. Also, even though the disk is perfectly flat, it appears to be warped upward behind the BH. This is because of the deflection of light rays by the gravity of the BH. (Based on Armitage \& Reynolds 2003; movie courtesy the authors)

Movie4.- Shows the variation of the fluorescent iron line in the simulation shown in Movie3. The inclination is held fixed at $80^{\circ}$ and the line emissivity is taken to be proportional to the local energy generation rate. Note that the line profile, shown at bottom right, varies rapidly as a result of turbulent fluctuations in the disk. The line extends from about $4 \mathrm{keV}$ on the left to about $7 \mathrm{keV}$ on the right, with a peak at around $6 \mathrm{keV}$. (Based on Armitage \& Reynolds 2003; movie courtesy the authors)

Movie5.- Simulation of a magnetic flux tube accreting onto a maximally rotating BH (Semenov et al. 2004). The light circle at the center represents the event horizon of the $\mathrm{BH}$, and 
the shaded region around it is the ergosphere. As the field line is drawn in by the gravity of the $\mathrm{BH}$, it is pulled forward azimuthally by the dragging effect of the $\mathrm{BH}$ spin. As a result, some of the plasma near the equatorial plane, shown in red, acquires negative energy as viewed from infinity. When this gas falls into the $\mathrm{BH}$, it effectively reduces the energy and angular momentum of the $\mathrm{BH}$. Correspondingly, electromagnetic and plasma energy is ejected along twin jets that move out parallel to the spin axis of the BH. Koide et al. (2002) named this the MHD Penrose process of extracting energy from a rotating BH. (Movie from Semenov et al. 2004, courtesy Brian Punsly)

Movie6. - Expanded view of the simulation shown in Movie5. Note the dramatic coiled magnetic field in the two outgoing jets. The jets are powered by the spinning BH via the MHD Penrose process. (Movie from Semenov et al. 2004, courtesy Brian Punsly) 


\section{Figure Captions}

Fig. 1.- [Upper panel] The red, blue and green spots show the relative locations of maser emitting clouds in the gas disk at the center of NGC 4258, as determined with radio interferometry. The black dot is the calculated position of the central supermassive BH. Emanating from the $\mathrm{BH}$ are the beginnings of twin jets. The dashed lines trace out a model of the disk. [Bottom panel] The dots show the line-of-sight velocities of the maser spots as a function of distance from the $\mathrm{BH}$ along the major axis of the disk. Note how perfectly the velocities

follow the Keplerian profile traced by the line. By fitting the velocities, the mass of the $\mathrm{BH}$ is inferred to be $3.5 \pm 0.1 \times 10^{7} M_{\odot}$. (Based on Greenhill et al. 1995; Herrnstein et al. 1998; image courtesy Lincoln Greenhill) 


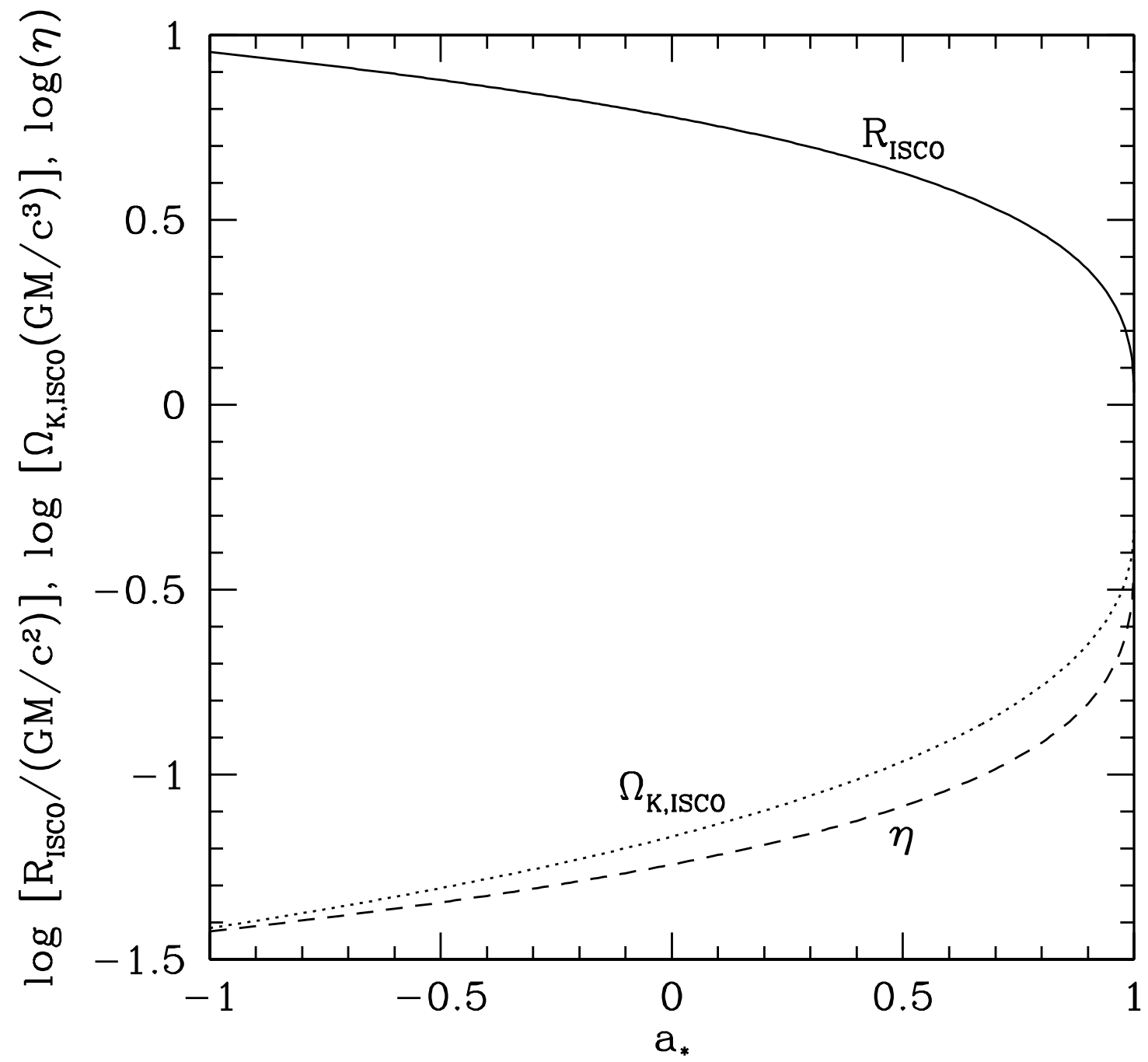

Fig. 2.- The radius of the innermost stable circular orbit $R_{\mathrm{ISCO}}$, the Keplerian frequency at this radius $\Omega_{\mathrm{K}, \mathrm{ISCO}}$, and the binding energy at this radius $\eta$, as functions of the $\mathrm{BH}$ spin parameter $a_{*}$. Positive values of $a_{*}$ imply that the $\mathrm{BH}$ corotates with the orbit and negative values mean that the $\mathrm{BH}$ counter-rotates. By measuring the quantity $R_{\mathrm{ISCO}} / M$ or $\Omega_{\mathrm{K}, \mathrm{ISCO}} M$ or $\eta$, one could estimate $a_{*}$. 


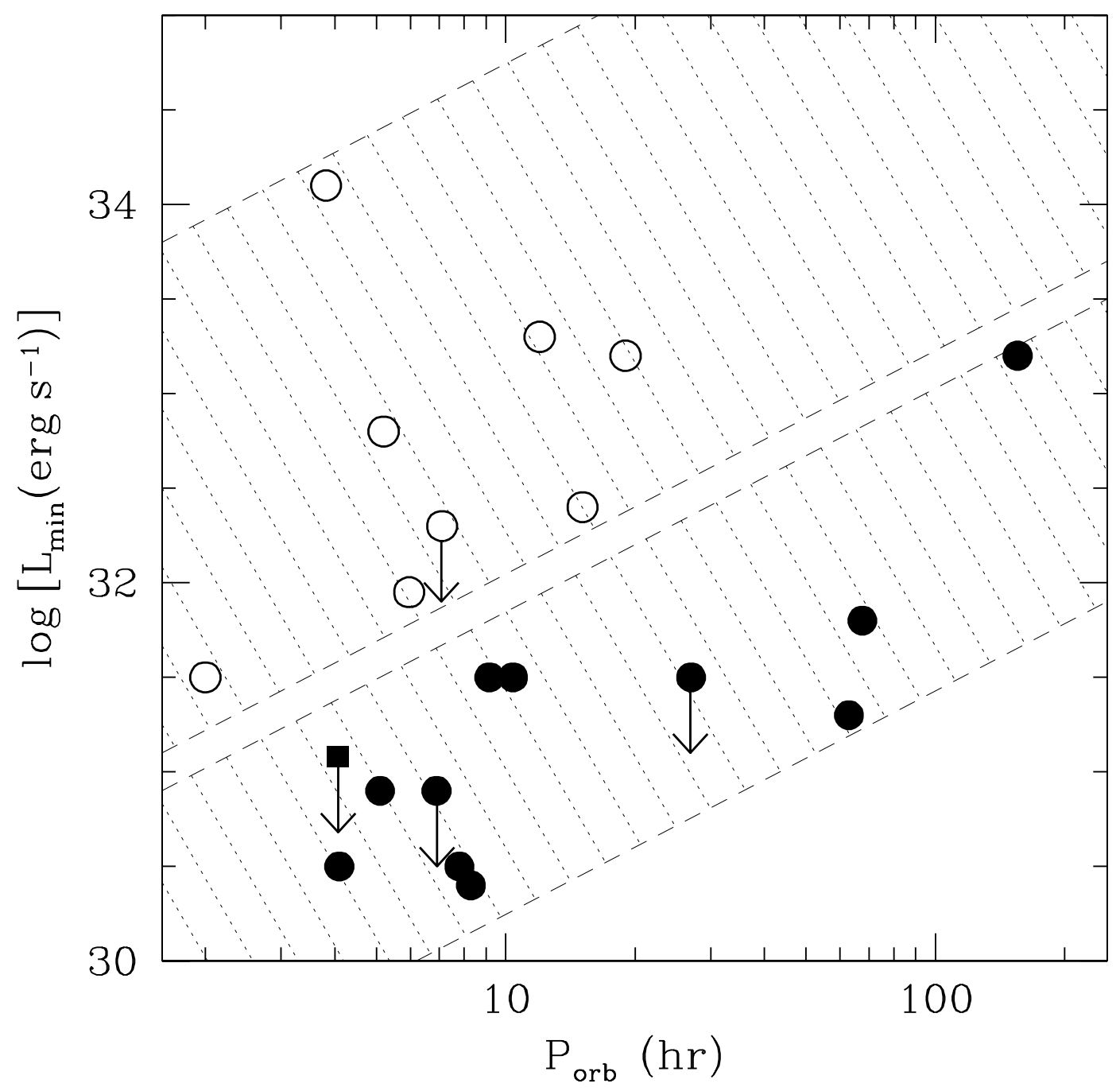

Fig. 3.- Quiescent luminosities $L_{\text {min }}$ of X-ray novae plotted against the orbital period $P_{\text {orb }}$ of the binaries. The filled symbols correspond to BH candidates, the open symbols to NSs, and arrows represent upper limits. The shaded bands are to guide the eye. At any given orbital period, the NSs as a group are a factor of $\sim 100$ brighter than the BH candidates. This difference may be interpreted as evidence that the $\mathrm{BH}$ candidates possess event horizons. (Taken from McClintock et al. 2004) 